\title{
Corrigendum: Retinal vessel caliber and its relationship with nocturnal blood pressure dipping status: the SABPA study
}

Wayne Smith, Nicolaas T Malan, Aletta E Schutte, Rudolph Schutte, Catharina MC Mels, Walthard Vilser and Leone Malan

Hypertension Research (2016) 39, 752; doi:10.1038/hr.2016.102

Correction to: Hypertension Research (2016) 39, 730-736; doi:10.1038/ hr.2016.48; published online 19 May 2016

After online publication of this article, the authors noticed an error in the affiliation of the list of authors.
The affiliation of Rudolph Schutte and Walthard Vilser was wrong. The error has now been rectified, and the article with correct authors' affiliations now appears in this issue. The html and online pdf versions have also been rectified. 